\title{
Analysis of Sparsely Populated Sets
}

\author{
Kiamran Radjabli* \\ Utilicast, La Jolla, California, USA. \\ *Corresponding author. Email: kradjabli@utilicast.com \\ Manuscript submitted April 25, 2016; accepted September 21, 2016. \\ doi: 10.17706/ijapm.2017.7.1.12-23
}

\begin{abstract}
Emptiness is usually considered to be absolute and equal to null or zero. This paper proposes to analyze conditions close to emptiness. A relaxation of abstract concepts of zero and emptiness is used to analyze sparsely populated sets. The proposed definition of emptiness is based on the Riemann's zeta function and depends on the perspective of the point from which emptiness is perceived. The new symmetric and asymmetric analytic continuations are proposed to resolve the asymptotic behavior of the zeta function near the pole. Different levels of infinity and emptiness are associated with "analytic convergence" using the asymmetric analytic continuation for the zeta function. The classifications of emptiness and infinity improve efficiency of data search in sparsely populated large sets, especially, when the data retrieval effort depends on the distance to remote data source.
\end{abstract}

Key words: Sparse, population, infinity, sets, zero, emptiness, classification, zeta function, deformation, search.

\section{Introduction}

The common understanding of "emptiness" is a domain or population with no elements. This is an abstract concept, which works very well for mathematical calculations. However, approaching absolute emptiness with no elements maybe just as strong of an abstraction as approaching infinity or approaching absolute zero. Do an absolute zero and infinity really exist or they are just the perception boundaries, because of the limits of our knowledge about the physical world, which is yet to be explored? For operating with extremely small and extremely large values, a new mathematical apparatus may need to be developed to obtain successful results. Some new research papers [1], [2] address the division by zero using the introduction of "nullity" $\Phi$ as a new number to model "undefined values." This approach defines a "transreal arithmetic" on the set of numbers that contains $\{-\infty,-1,0,1,+\infty, \Phi\}$ as a proper subset. Also, the modern mathematics attempts to address the properties of zero and infinity through an introduction of "surreal" number system, which is an arithmetic continuum containing the real numbers as well as infinite and infinitesimal numbers, respectively larger or smaller in absolute value than any positive real number [3]. Adding one positive infinity with another positive infinity is usually considered to result in yet another infinity, because the infinity indicates that limits "grow beyond any number." However, intuitively, we understand that this is a level of abstraction that may need to be quantified, and the resulting infinity may have a higher "level" of infinity than the two infinite components that form the sum. Determination of infinity's level requires classification of infinities. In a similar way, we can consider that domains in infinite space with extremely dispersed elements may have different degree of "emptiness", which can be distinguished and manipulated at the level of extremely small values. When assumed zero value is not 
precisely zero for very small values, then a simple measure and a classification of emptiness are needed to operate with "quasi-empty" domains.

\section{Measure of Emptiness}

A system may contain complexities, which are not effectively captured by a model of the system, and thus a zero value in the model may not be a precise zero. Let us assume that there is a very small quasi-zero value $\varepsilon_{0}$, which is considered so close to an absolute zero value 0 , that for all practical purposes in the physical world it can be treated as zero. The value $\boldsymbol{\varepsilon}_{\mathbf{0}}$ represents an "imprecise zero" as the low limit of the study environment, where extremely small value can work just as fine as an absolute zero in terms of getting realistic results in an infinite domain.

The broad understanding of zero encompasses different categories of zero, e.g. a zero scalar value, a zero-magnitude vector, an empty set without elements, a region of space with zero volume, a null as the lack of an element. The focus of this analysis are sets of coordinates with very small number of dispersed points in extremely large space domain approaching infinity, and subsequently, the space occupancy approaching zero. The non-absolute nature of emptiness is understood as reaching an "imprecise zero" value of "quasi-emptiness." The number of points and their coordinates in space determine the emptiness. Therefore, the measure of space emptiness is relative to the view point, from which the emptiness is perceived.

Let us consider a sparsely populated set of points in infinite space domain. For the determination of "emptiness", we propose to evaluate the amount of non- $\emptyset$ values in the set from a chosen study "view" point in space. The further the non- $\emptyset$ values are from the "view" point, the less is their contribution to filling the space domain of the set. For two-dimensional analysis, Euclidean distances from the "view" point coordinates $\left(\mathbf{x}_{\mathbf{0}}, \mathbf{y}_{\mathbf{0}}\right)$ to the point with coordinates $\left(\mathbf{x}_{\mathbf{A}}, \mathbf{y}_{\mathbf{A}}\right)$ can quantify how far away the non- $\emptyset$ value is located in the set.

$$
D\left(x_{A}, y_{A}\right)=\frac{\sqrt{\left(x_{A}-x_{0}\right)^{2}+\left(y_{A}-y_{0}\right)^{2}}}{\sqrt{x_{0}^{2}+y_{0}^{2}}}
$$

The distance D normalized to the "view" point coordinates depends on the coordinates of the "view" point and can represent a simple measure of relative emptiness from that "view" point to the next non- $\emptyset$ value. The following formula is proposed to find the measure of "quasi-emptiness" in a two-dimensional domain, but can be generalized for a multi-dimensional space domain.

$$
E=\sum_{n=1}^{N_{\varepsilon}} \frac{D_{0}}{D_{n}}=\sum_{n=1}^{N_{\varepsilon}} \frac{D_{0}}{\sqrt{\left(x_{n}-x_{0}\right)^{2}+\left(y_{n}-y_{o}\right)^{2}}}
$$

where $\mathbf{n}$ is the sequential number of the non- $\emptyset$ subset; $\mathbf{D}_{\mathbf{n}}$ is the distance to a non- $\emptyset$ subset number $\mathbf{n} ;\left(\mathbf{x}_{\mathbf{n}}, \mathbf{y}_{\mathbf{n}}\right)$ are the coordinates of the non- $\emptyset$ subset number $\mathbf{n} ;\left(\mathbf{x}_{\mathbf{0}}, \mathbf{y}_{\mathbf{0}}\right)$ are the coordinates of a "view" point. In formula (2), it is assumed that the distances are sorted in their incremental order, i.e. $\mathbf{D}_{\mathbf{n + 1}}>\mathbf{D}_{\mathbf{n}}$. The normalization factor will be dropped further below assuming that it is incorporated in normalized coordinates and the pertinent distance $\mathbf{D}_{\mathbf{n}}$. The summation over number $\mathbf{n}$ is performed on all components of the set until we reach the $\mathbf{N}_{\varepsilon}$, which corresponds to the circular border $(\mathbf{X}, \mathbf{Y})$ determined by quasi-zero value $\boldsymbol{\varepsilon}_{\mathbf{0}}$, i.e.

$$
\left(X-x_{0}\right)^{2}+\left(Y-y_{o}\right)^{2}=\frac{x_{0}^{2}+y_{0}^{2}}{\varepsilon_{0}^{2}}
$$

\section{Emptiness Classification}

We propose to distinguish "quasi-empty" sets defined in space domain using the Riemann zeta-function 
[4], [5] for an integer argument s

$$
\zeta(\mathrm{s})=\sum_{\mathrm{n}=1}^{\infty} \frac{1}{\mathrm{n}^{\mathrm{s}}}
$$

The value of $\zeta(2 s)$ for even integers is known [4], [5] to be calculated using Bernoulli's numbers B [6], [7].

$$
\zeta(2 s)=\frac{(2 \pi)^{2 s}(-1)^{(s+1)} B_{2 s}}{2(2 s) !}
$$

If distances $\mathbf{D}_{\mathbf{n}}$ for each next element $\mathbf{n}$ of the set grow as fast as $\mathbf{n}^{\mathbf{s}}$ then we can consider that the growth of distances between elements of the set are of the same power as $\mathbf{n}^{\mathbf{s}}$. Thus, the density of elements in a study set can be compared to the density of $\mathbf{n}^{s}$.

For $s=1$, the zeta function diverges, i.e. $\zeta(1)=\infty$. Therefore, we introduce the following new "symmetric analytic continuation" function:

$$
\theta(s)=\zeta(s)+\zeta(2-s)
$$

where, if $s=1, \theta(s)=\lim _{\Delta s \rightarrow 0} \zeta(1+\Delta s)+\zeta(1-\Delta s)$

The function $\boldsymbol{\theta}(\mathbf{s})$ and its components are shown on Fig. 1 with smoothed curves and interpolated values. The analytic continuation of the zeta function $\zeta(\mathbf{s})$ for $s<1$ is also shown smoothed and without trivial zeros for even integer $\mathbf{s}$ values. The formula for calculation of the analytic continuation was initially proposed by Euler and later generalized using Bernoulli numbers:

$$
\zeta(-m)=(-1)^{m} \frac{B_{m+1}}{m+1}
$$

for $m=0,1,2,3, \ldots$

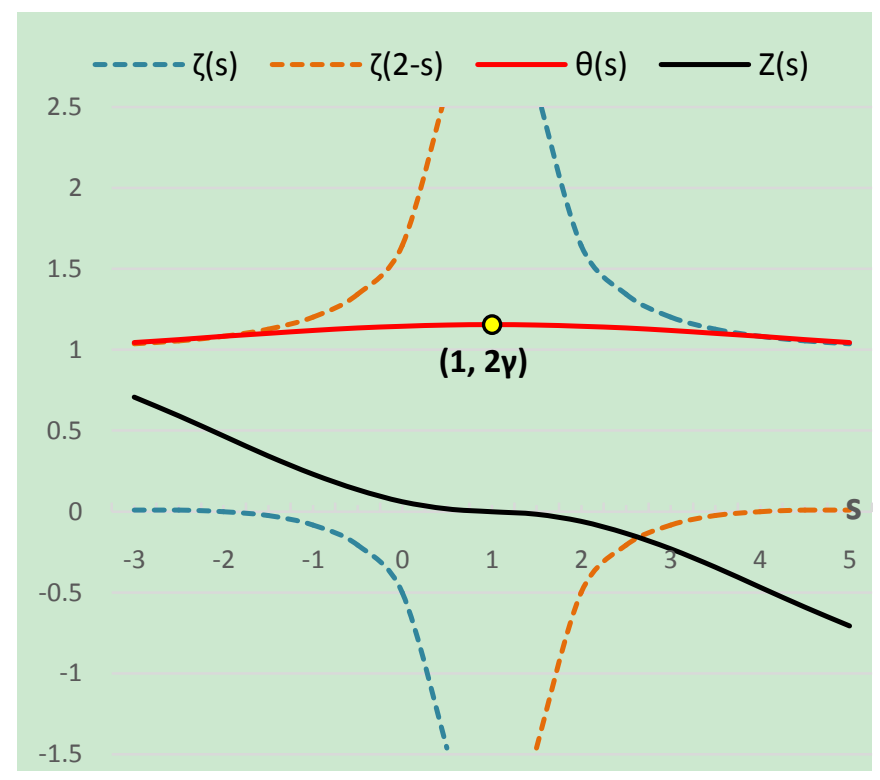

Fig. 1. Symmetric $\theta(\mathrm{s})$ and asymmetric $Z(\mathrm{~s})$ analytic continuations of $\zeta(\mathrm{s})$.

Analytic continuation on the sums (4) is illustrated with a smoothed version of the sums and its cutoff function with the appropriate properties. As shown on Fig. 2, analytic continuation is visualized as an 
intersection of the smoothed function with the vertical axis. For $s=0$, that smoothed version of the sum is a linear function $y(n)=n-1 / 2$, and the intersection with the vertical axis $y(0)=\zeta(0)=-1 / 2$. A similar approximation of summations exist for $s<0$. For $s=1$, the intersection does not exist and the approximated function $\mathbf{y}(\mathbf{n})$ is diverging in both directions (i.e. for $n \rightarrow 0$ and $n \rightarrow \infty$ ).

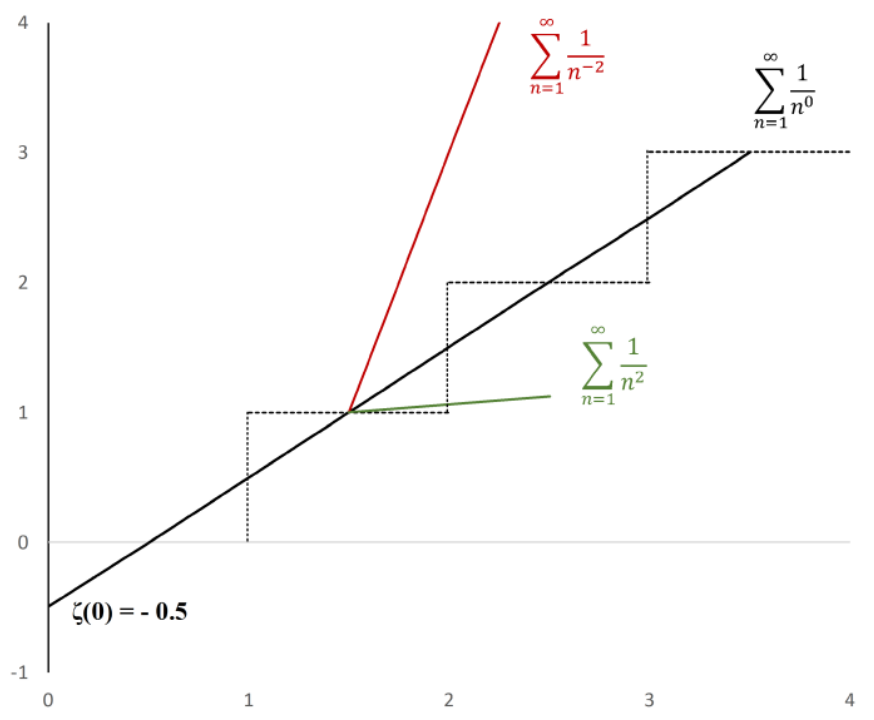

Fig. 2. Visualization of analytic continuation for zeta function.

Although $\zeta(1)$ does not exist, it is possible to extract the Euler-Mascheroni constant $\boldsymbol{\gamma}$ for $s=1$ [6], [7] as shown in formula (8) below.

$$
\zeta(s)=\frac{1}{1-s}+\gamma+f(s-1)
$$

where $f(s-1) \rightarrow 0$, as $s \rightarrow 1$, and

$$
\gamma=\lim _{n \rightarrow \infty}\left(\sum_{k=1}^{n} \frac{1}{k}-\ln (n)\right)^{n}=0.5772 \ldots
$$

Then, using equation (6) in (8) for $s=1$, we can establish

$$
\theta(1)=2 \gamma
$$

The derivative of $\boldsymbol{\theta}(\mathbf{s})$ for small $\mathbf{s}$ is determined using the zeta function derivative formula

$$
\zeta^{\prime}(s)=-\frac{1}{s-1}+\sum_{n=0}^{\infty}(-1)^{n} \frac{\gamma_{n}}{n !}(s-1)^{n}
$$

where $(-1)^{n} \frac{\gamma_{n}}{n !}$ are Stieltjes constants, and $\gamma_{0}=\gamma$.

Then, using definition (6) for $\boldsymbol{\theta}(\mathbf{s})$ and formula (10), we can conclude that

$$
\theta^{\prime}(1)=0
$$




$$
\theta(1)=\theta_{\max }=2 \gamma
$$

The function $\boldsymbol{\theta}(\mathbf{s})$ is defined based on the zeta function, but unlike the latter, $\boldsymbol{\theta}(\mathbf{s})$ does not have apole, it is continuous, and has no trivial zeroes for all $\mathbf{s}$. Essentially, $\boldsymbol{\theta ( \mathbf { s } )}$ is the absolute difference of the zeta function for $s>1$ and its analytic continuation for $s<1$. The growth of the zeta function for $s>1$ and the growth of the analytic continuation for $s<1$ near the pole $(s=1)$ are of the same magnitude, and their difference converge to the value equal to two times that of the Euler constant $\boldsymbol{\gamma}$. Usually the sum of positive and negative infinity in considered to be indeterminate (unless the continuum of "surreal numbers" is considered [3]). The function $\boldsymbol{\theta ( s )}$ for $s=1$ is the case when "adding" two infinities of opposite sign is clearly interpreted by a finite value.

We will define the "asymmetric analytic continuation" Z(s) functionas follows:

$$
Z(s)=\frac{2 \gamma-\theta(s)}{2 \gamma-1} \operatorname{sign}(1-s)
$$

The "asymmetric analytic continuation" Z(s) can serve as a progression rate or "strength" of the $\zeta(\mathbf{s})$ zeta function series (4) and the emptiness.

In general, distances $\mathbf{D}_{\mathbf{i}}$ do not tend to progress precisely at the rate of $\mathbf{n}^{\mathbf{s}}$ for each consecutive distance calculation from one element to the next element of a set. However, we can "estimate" the $\mathbf{D}_{\mathbf{i}}$ trend by associating it with the $\boldsymbol{\zeta ( \mathbf { s } )}$ zeta function series. A corresponding value $\mathbf{s}$ of the associated zeta function can represent a "density" of the set with $\mathbf{D}_{\mathbf{i}}$ distances, and $\mathbf{Z}(\mathbf{s})$ can represent the progression strength of elements in the set.

Thus, we propose to classify infinite sets by relating values of emptiness $\mathbf{E}$ and $\zeta(s)-1$ as follows:

$$
\begin{gathered}
\text { Non Empty: } E+1>\zeta(2)=\frac{\pi^{2}}{6} \\
\text { Empty: } E+1 \leq \zeta(2)=\frac{\pi^{2}}{6}
\end{gathered}
$$

For very large finite sets, a similar approach is exercised by assessing a value of emptiness $\mathbf{E}$ for all components of the set until we reach the circular border determined by the quasi-zero value $\varepsilon_{0}$ in equation (3). If emptiness $\mathbf{E}$, calculated within the quasi-zero value border is remaining greater than $\zeta(2)-1$, then we consider the set non-empty. Otherwise the set is called "empty." After associating the summation of distances with the $\boldsymbol{\zeta ( \mathbf { s } )}$ zeta function for $s \geq 2$ and establishing equivalent $\mathbf{s}$, an asymmetric analytic continuation $\mathbf{Z}(\mathbf{s})$ is calculated to determine the measure of the density in the set. This function can be used to translate emptiness into a value defined for any $\mathbf{s}$, where $\mathbf{Z}(\mathbf{s})$ for extremely high emptiness approaches minus one, and $\mathbf{E}$ is approaching zero.

It is also possible to elaborate on the "non-empty" quality and establish a measure of the set's population in terms of high density for $s \leq 0$. The asymmetric analytic continuation $\mathbf{Z ( s )}$ distinguishes the sets with very high compression and translate that compression into a value defined for any $\mathbf{s}$, where $\mathbf{Z}(\mathbf{s})$ for extremely high compressions approach plus one, and $\mathbf{E}$ is approaching infinity.

All formulas above are based on the Euclidean distance. In a three-dimensional domain, it is more applicable to use non-Euclidean's geometry, like the elliptic Riemann-Finsler [8]-[11] geometry. The latter allows applying the curvature geodesic and sense of direction to the distance measured from one point to another. The concepts and classifications established here with Euclidean geometry can be applied to similar analysis using Riemann-Finsler calculations of distance, but the details of such a presentation is 
beyond the scope of this paper.

\section{Infinity Classification}

The classic definition of infinity is "a number greater than any assignable quantity or countable number." Let us assume that there can be different levels of infinity even if the actual quantity is undefined and diverging. An unassignable quantity can be potentially evaluated on its quality of largeness compared to other unassignable quantities. For example, if $x \rightarrow \infty$, then, the functions $f_{1}(x)=a x$ and $f_{2}(x)=b x^{2}$ also approach infinity, but with very different intensity. For a very large argument $\boldsymbol{x}$ approaching infinity, the function $\boldsymbol{f}_{2}$ is much greater than $\boldsymbol{f}_{\mathbf{1}}$, as $\boldsymbol{x}$ is greater than any finite values of $\boldsymbol{a}$ and $\boldsymbol{b}$. Therefore, the functions $\boldsymbol{f}_{\mathbf{1}}$ and $\boldsymbol{f}_{\mathbf{2}}$ have no intersection for $x \rightarrow \infty$ and approach different quality levels of infinity or effectively belong to different classes of infinity. The intensity of the function's growth or its derivative becomes a determining factor of the infinity level that function can approach. Let's associate the infinity of the power function $f=a x^{s}$ with its integral, and subsequently, with the corresponding summation of the zeta function.

$$
\lim _{x \rightarrow \infty} a x^{s} \Rightarrow \sum_{n=1}^{\infty} \frac{1}{n^{-s}} \Rightarrow Z(-s)
$$

In spite of the fact that the infinity of the function does not have any directly assignable quantity or countable number, the analytical association with the zeta function provides a virtual finite value that can be assigned to the intensity level of the divergence, which can qualify the class of divergence. For a power function, the infinity class $\mathbf{s}$ is considered to be "analytically converging" to the value of $\mathbf{Z}(\mathbf{s})$.

\section{Operations on Quasi-Empty Sets}

In addition to the proposed measure $\mathbf{E}$ of "quasi-emptiness" and measure $\mathbf{Z}(\mathbf{s})$, we propose to assign a quality class $\mathbf{K}$ of emptiness to a set using the zeta function. The class of emptiness is equal to an integer value $\mathbf{K}>0$, such that

$$
\zeta(K+2)<E+1 \leq \zeta(K+1)
$$

For a "non-empty" set, the class $\mathbf{K}=0$. The higher class corresponds to a higher degree of emptiness, and for $K \rightarrow \infty$, emptiness $E \rightarrow 0$.

The following global topological deformations can represent operations on quasi-empty sets with finite or infinite number of elements: Expansion, Contraction, Warping, and Twisting.

It is possible to analyze other global deformations, e.g. partial morphing, swirling, taper and the compound of several global deformations, which are beyond the scope of this paper's analysis.

The deformation strength, or "level" of deformation $\mathbf{L}$, is defined here in relation with emptiness as a change of class $\mathbf{K}$ after applying the deformation.

$$
\begin{array}{r}
E \leq \zeta(s)-1 \\
E_{(D F)} \leq \zeta(s \pm L)-1
\end{array}
$$

where $\mathbf{E}$ and $\mathbf{E}_{(\mathrm{DF})}$ are emptiness of the original and deformed sets and $\mathbf{S}$ and $\mathbf{L}$ are positive integer values.

Expansion is an operation that evenly "stretches" the quasi-empty set to change its quality from one class of emptiness to a higher class, i.e. operation expands a set to the $+\mathbf{L}$ higher class.

Contraction is an operation that evenly "shrinks" the quasi-empty set to change its quality from one class 
of emptiness to a lower class, i.e. contracts a set to the -L lower class. The lowest level of contraction is the zero class of the non-empty set.

A uniform linear expansion/contraction results in effective global scaling deformation, which results in getting all coordinates of the elements scaled proportional to the same value $\mathbf{r}$. Then, the expanded/contracted emptiness

$$
E_{(r)}=\sum_{n=1}^{N_{\varepsilon}} \frac{1}{\sqrt{\left(r x_{n}-x_{0}\right)^{2}+\left(r y_{n}-y_{o}\right)^{2}}}
$$

Let us interpret the global scaling deformation of coordinates in the set as an equivalent L-level "expansion/contraction" in a zeta function by the following formula:

$$
\zeta(s \pm L)=1+\sum_{n=2}^{\infty} \frac{1}{r n^{s}}
$$

Then, based on formula (4) and (20), the value $\mathbf{r}$ resulting in level $\mathbf{L}$ expansion/contraction is presented as

$$
r(L)=\frac{\zeta(s)-1}{\zeta(s \pm L)-1}
$$

Warping is an operation that unevenly "bends" the quasi-empty set to change its quality only in specific parts of the set and results in the change of emptiness class. An example of warping on Fig.3 shows small circles as non-empty coordinates on the warped surface.

If we warp the one-dimensional domain set in two dimensions and introduce a second coordinate $\mathbf{y}$, then we can reduce the effective distance between x-coordinates calculated in two dimensions. In the one-dimensional domain, the distances remain the same, but in the two-dimensional domain, the warping results in getting the distance reduced by curving the space. The following formula calculates the length $\mathbf{D}_{\mathbf{L}}$ of a curve from $\mathbf{x}_{\mathbf{i}}$ to $\mathbf{x}_{\mathbf{j}}$.

$$
D_{L}=\int_{X_{i}}^{X_{j}} \sqrt{1+\left(\frac{d y}{d x}\right)^{2}} d x
$$

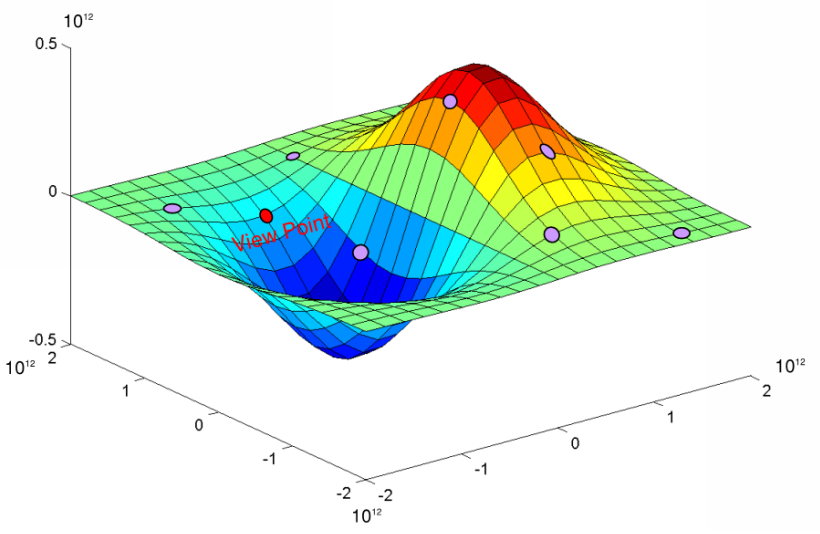

Fig. 3. Warping of quasi-empty set.

For illustration simplicity, let us assume that the curve is a half circle bending in $\mathbf{y}$ coordinate, which is referred further as warping. It is shown on Fig.4 how the original distance $\mathbf{x}_{\mathbf{j}}-\mathbf{x}_{\mathbf{i}}$ between point $\mathbf{i}$ with 
coordinate $\mathbf{x}_{\mathbf{i}}$ and point $\mathbf{j}$ (of a non- $\emptyset$ subset) with coordinate $\mathbf{x}_{\mathbf{j}}$ is reduced to a new distance $\mathbf{x}_{\mathbf{j}(w)}-\mathbf{x}_{\mathbf{i}}$ for a simple half-circular warping with radius $\mathrm{R}$, such that the length of the warped half circle is equal the original distance $x_{j}-x_{i}$

$$
\left(x_{j}-x_{i}\right)=\pi R
$$

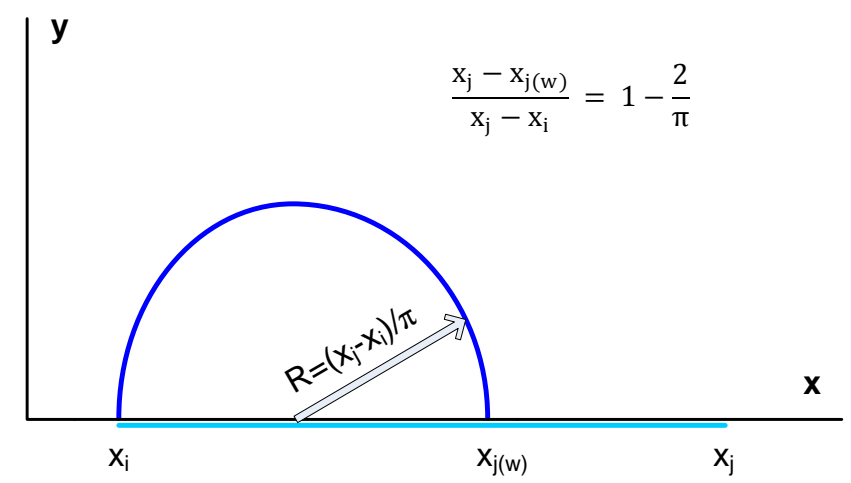

Fig. 4. Simplified half-circular warp deformation.

The new warped distance

$$
D_{w}=x_{j(w)}-x_{i}=2 R
$$

Thus, for the half circle warp the distance $\mathbf{x}_{\mathbf{j}}-\mathbf{x}_{\mathbf{i}}$ is effectively reduced in two-dimensional domain by

$$
x_{j}-x_{j(w)}=\left(x_{j}-x_{i}\right)\left(1-\frac{2}{\pi}\right)
$$

The change of coordinates determined by warp $\mathrm{W}$ is calculated by the following formula:

$$
W=1-\frac{D_{w}}{D}=1-\frac{x_{j(w)}-x_{i}}{x_{j}-x_{i}}
$$

where $0<\mathrm{W}<1$

In case of very high deformation, warp approaches one and the warped distance approaches zero, i.e. $\mathrm{W} \rightarrow 1$ and $\mathrm{D}_{\mathrm{w}} \rightarrow 0$, while $\mathrm{x}_{\mathrm{j}(\mathrm{w})}$ is approaching to $\mathrm{x}_{\mathrm{i}}$. In case of a very low deformation, warp approaches zero and the warped distance approaches the non-warped distance, i.e. $W \rightarrow 0$ and $D_{w} \rightarrow D$, while $x_{j(w)}$ is approaching $\mathrm{x}_{\mathrm{j}}$.

We can represent a set with the warp or bend between points $\mathbf{m}_{\mathbf{1}}$ and $\mathbf{m}_{\mathbf{2}}$ using formula (2) for quasi-emptiness.

$$
E_{(w)}=\sum_{n=1}^{m_{1}} \frac{1}{D_{n}}+\sum_{n=m_{1}+1}^{m_{2}} \frac{1}{D_{n(w)}}+\sum_{n=m_{2}+1}^{N_{\varepsilon}} \frac{1}{D_{n}}
$$

The warp results in a reduction of emptiness, which is equal to the difference between the non-warped and warped emptiness of the set.

$$
\Delta E_{(w)}=E_{(w)}-E=\sum_{n=m_{1}+1}^{m_{2}}\left(\frac{1}{D_{n(w)}}-\frac{1}{D_{n}}\right)>0
$$


The warp and subsequent reduction of emptiness may change the emptiness class. The comparison of the difference $\Delta \mathbf{E}_{(\mathrm{w})}$ and change of corresponding zeta function

$$
\Delta \zeta_{L}=\zeta(\mathrm{s}-\mathbf{L})-\zeta(\mathrm{s})
$$

can determine the $\mathbf{L}$ level of the warp in terms of emptiness.

Using definition of warp (25), for a warp occurring between two consecutive points, i.e. $m_{2}=m_{1}+1$

$$
\Delta E_{(w)}=\frac{1}{D_{n(w)}}-\frac{1}{D_{n}}=\frac{W}{D_{n}(1-W)}=\Delta \zeta_{L}
$$

Then, based on formulas (30) and (31), the following is the formula for the warp resulting in level $\mathbf{L}$ contraction:

$$
W(L)=\frac{D_{n}[\zeta(\mathrm{s}-\mathbf{L})-\zeta(\mathrm{s})]}{1+D_{n}[\zeta(\mathrm{s}-\mathbf{L})-\zeta(\mathrm{s})]}
$$

Twisting is an operation that modifies several coordinates, but keeps other coordinates unchanged. The following formula can represent a $\mathbf{z}$-axis twisting of $\mathbf{x}, \mathbf{y}, \mathbf{z}$ coordinates into new coordinates $\mathbf{x}_{\mathbf{t}}, \mathbf{y}_{\mathbf{t}}, \mathbf{z}_{\mathbf{t}}$ in three-dimensional space

$$
\begin{gathered}
x_{T}=x f_{T}(z) \\
y_{T}=y f_{T}(z) \\
z_{T}=z
\end{gathered}
$$

The function $\mathbf{f}_{\mathrm{T}}(\mathbf{z})$ determines the actual character and intensity of the twist, e.g. for the $360^{\circ}$ twist on the interval $\mathrm{z}_{\mathrm{n}}<\mathrm{z}<\mathrm{z}_{\mathrm{m}}$, the function $f_{T}(\mathrm{z})=\frac{z_{m}-z}{z_{m}-z_{n}}$. Twisting operation does not result in the change of an emptiness class, but can be compounded with other deformations described above, which do affect emptiness.

\section{Application of Emptiness in Quasi-Empty Sets}

Now that we have established the basis for classification of sparsely populated sets with an infinite number of elements, let us illustrate how the concept of quasi-emptiness is applied to the search in "quasi-empty" sets. We assume that there is a large number of multisets $\mathbf{P}$, which are sparsely populated with subsets $\mathbf{A}_{\mathbf{i}}$. The "time-effort" of analyzing components of the set is increasing with the distance from the beginning of each set. Thus, the father the element is from the "view" point the longer the processing time and the larger the effort it takes to fetch the information about that element. Any element in the set can potentially contain valuable information, but a balance needs to be reached between the effort of the information extraction and the value of that information.

Each subset $\mathbf{A}$ consists in its turn of $\mathbf{- 1}, \mathbf{0}, \mathbf{+ 1}$, and $\mathbf{U}$, where $\mathbf{U}$ is "instability", represented by infinite summation of $\mathbf{+ 1}$ and $\mathbf{- 1}$ as per formula (36). The result of the summation is either $\mathbf{1}$ or $\mathbf{0}^{1}$.

\footnotetext{
${ }^{1}$ Diverging series of "Ramanujan summation" [11] are associated with a single finite number, e.g. $\sum_{\mathrm{n}=0}^{\infty}(-1)^{\mathrm{n}}=\zeta(0)=-\frac{1}{2}$.
} However, in the example of search analysis, the $U$ summation is still considered diverging and indeterminate. 


$$
U_{b}=1-1+1-1+1-1+1 \ldots
$$

The instabilities $\mathbf{U}$ are randomly spread in subsets $\mathbf{A}_{\mathbf{i}}$, and the objective of the search is to find as many instabilities as possible in an allocated time period. We also add a special condition that instability is detected only when the unstable element is viewed several times, because it oscillates in the range of $\mathbf{1}$ to $\mathbf{0}$ each time it is viewed. The existence of $\mathbf{U}$ is rare enough to occur, and a subset $\mathbf{A}_{\mathbf{i}}$ may have either one $\mathbf{U}$ or none.

In order to make the search algorithm more efficient compared to brute-force calculations, the following procedure is executed using quasi-empty definitions and classifications.

1) Establish small quasi-zero value $\boldsymbol{\varepsilon}_{\mathbf{0}}$

2) Establish the classification $\mathbf{L}_{\mathbf{P}}$ for all multisets $\mathbf{P}$ as per formula (17). Segregate empty and non-empty multisets. Start the search analysis from the non-empty sets and then proceed in the increasing class of emptiness to ensure that the most populated domains are analyzed with higher priority.

3) Examine $\mathbf{A}_{\mathbf{i}}$ only in the multisets $\mathbf{P}$ of class $\mathbf{L}_{\mathbf{P}}$ using quasi-zero value $\boldsymbol{\varepsilon}_{\mathbf{0}}$ within the extent of search border

a. For each $\mathbf{A}_{\mathbf{i}}$ in $\mathbf{P}$, establish its own set classification $\mathbf{L}_{\mathbf{A}}$ and at the same time "memorize" the total "checksum" by adding non-zero elements in the set.

b. For subsets $\mathbf{A}_{\mathbf{i}}$ of class $L_{A}=0$, re-open the subset and verify the change of "checksum" in order to detect the existence of $\mathbf{U}$.

c. If checksum has not changed, then continue searching in the next subset.

d. If checksum has changed, then examine all non-zero elements and find coordinates of $\mathbf{U}$ in the subset $\mathbf{A}_{\mathbf{i}}$.

4) Repeat the previous steps for all multisets until the allocated search time expires or satisfactory number of objects are found.

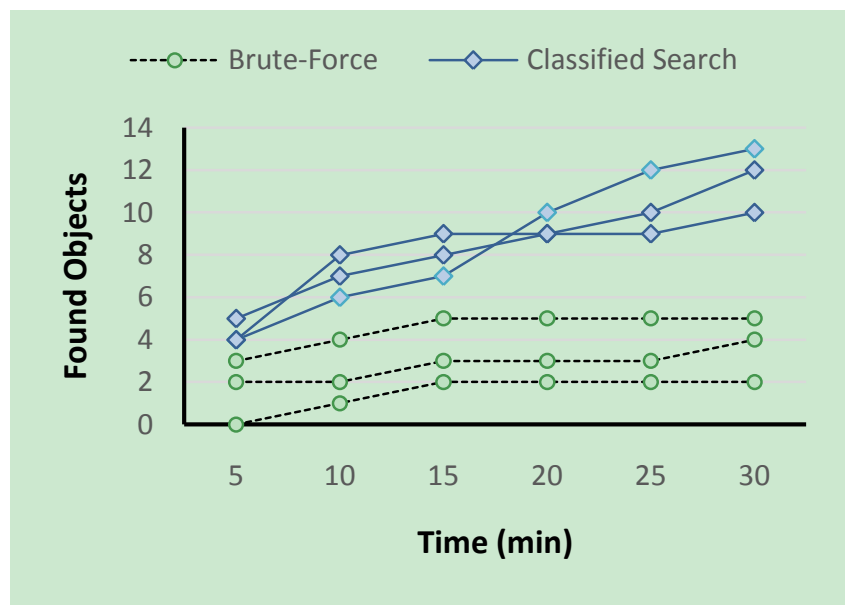

Fig. 5. Comparison of results for the search of information in sparsely populated large sets.

The above algorithm was evaluated using simplified software model developed specifically for the analysis of search efficiency. The model simulates two dimensional space coordinates as a very large matrix of quadrants. The data retrieval effort is simulated by a time delay proportional to the distance between the reference point and the location of information. Also, the detection of a non-zero value takes longer time than a detection of zero or null value. The following are parameters of the simulation: 10,000 sparsely 
populated multisets $\mathbf{P}$, population of 1000 subsets $\mathbf{A}$ distributed in multisets $150 \%$ randomly distributed and $50 \%$ distributed with exponentially increasing coordinates in each multiset), and 20 instabilities $\mathbf{U}$ with totally random distribution. The objective of the search was to find as many the instabilities $\mathbf{U}$ in the allocated period of time. The Fig. 5 shows the results of comparison between brute-force sequential search and the search with pre-processing classification multisets $\mathbf{P}$. The comparison demonstrates that the sequential approach finds less objects of interest in allocated time, because the sequential scan sometimes gets "trapped" in the populations that have no valuable information. The classification pre-processing followed by search retrieves more objects of interest as it has prior "intelligence" of populations with "non-empty" quality. The search moves from one "non-empty" population to another upon achieving the pre-established border of the population spread in space.

\section{Conclusions}

The objective of this research is analysis of non-absolute nature of infinity, zero, and emptiness. The proposed approach of "imprecise zero" and association with Riemann's zeta function provide a possibility to measure the emptiness of sparsely populated sets of coordinates, which contain infinite number of elements. The following bullets summarize the results of this research:

1. The measure of emptiness is introduced using distances to the set's elements relative to the "view" point.

2. Infinity is classified by the quality of the diverging intensity. Emptiness is classified by the quality of the space density. The classifications of "quasi emptiness" allows to define operations in the form of global deformations, such as expansion, contraction, warp, and twist.

3. New symmetrical and asymmetrical analytic continuations of Riemann's zeta function are proposed to "resolve" its asymptotic infinities at the pole. The asymmetrical analytic continuation provides a possibility to evaluate and assign a value to the degree of emptiness and infinity.

4. The measure of emptiness establishes a basis for a more efficient information search in infinite and sparsely populated domains, where the information extraction effort depends on the coordinates of the elements in the set. Analyzing classification of infinite sets prior to performing the information search, provides a practical way to the extraction of information, which is faster than brute-force sequential analysis of information in infinite domains. The pre-processing and evaluation of information, which is natural for human approach to search problems is applicable to computer analysis.

\section{References}

[1] Reis, T., Anderson, J., \& Transreal, C. IAENG International Journal of Applied Mathematics, 45(1), 51-63.

[2] Anderson, J., Völker, N., Adams, A. (2015). Perspex machine VIII: Axioms of transreal arithmetic. Proceedings of SPIE: Vol. 6499, Vision Geometry XV.

[3] Ehrlich, P. (2012). The absolute arithmetic continuum and the unification of all numbers great and small. The Bulletin of Symbolic Logic, 18(1), 1-45.

[4] Abramowitz, M., \& Stegun, I. A. (1972). Riemann zeta function and other sums of reciprocal powers. Handbook of Mathematical Functions with Formulas, Graphs, and Mathematical Tables (pp. 807-808).

[5] Adamchik, V. S., \& Srivastava, H. M. (1998). Some Series of the Zeta and Related Functions. Analysis 18 (pp. 131-144). 
[6] Abramowitz, M., \& Stegun, I. A. (1972). Bernoulli and euler polynomials and the euler-maclaurin formula. Handbook of Mathematical Functions with Formulas, Graphs, and Mathematical Tables (pp. 804-806).

[7] Arfken, G., et al. (1985). Mathematical Methods for Physicists (pp. 327-338). Orlando, FL: Academic Press.

[8] Bao, D., Bryan, R., Chern, S., \& Shen, Z. (2010). A Sampler of Riemann-Finsler Geometry.

[9] John, M. L. (1997). An introduction to curvature. Riemannian Manifolds, Graduate Texts in Mathematics.

[10] Michael, S. (1979). A comprehenssve introduction to differential geometry.

[11] Bruce, C. (1939). Ramanujan's Notebooks, Ramanujan's Theory of Divergent Series.

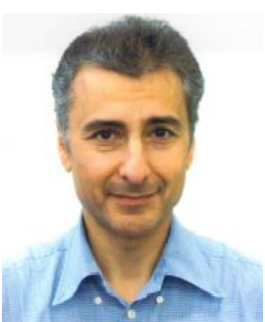

Kiamran Radjabli was born in 1959 in Baku, Azerbaijan. He earned his master of science and the doctorate degrees in electrical engineering from Moscow Power Engineering Institute. He is a professional engineer licensed in Ontario, Canada. He provided consulting services to a number of electrical utilities in North America and abroad. He is carrying out research in the area of sparsely populated sets, conditions close to emptiness, and infinite space domains. Kiamran has over 20 years of experience in control systems, software development, and real time applications. 